\title{
Home inotrope treatment for intractable heart failure following heart transplantation
}

\author{
S W Dubrey, N Banner
}

\begin{abstract}
A 49 year old man developed intractable heart failure three years after undergoing heart transplantation. Coronary angiography showed no evidence of graft vascular disease. An initial cardiac biopsy identified one episode of rejection which responded to augmented immunosuppressive treatment. The patient became inotrope dependent and has now survived at home for 22 months using an ambulatory delivery system for intravenous adrenaline (epinephrine), without significant complications. There has been a noticeable improvement in symptoms and left ventricular systolic performance, both clinically and as seen through echocardiographic and radiographic examination. This improvement was substantiated by the results of cardiac catheterisation, which showed a return to normal left ventricular filling pressure and cardiac output. The case is noteworthy because this treatment has allowed a patient who otherwise would have been hospital bound to return to the community. With the current shortage of organs, he would have been unlikely to receive a second transplant. The clinical features and outcome, and social, medicolegal, and financial issues are discussed.

(Heart 1999;82:248-252)
\end{abstract}

Keywords: transplantation; heart failure; inotropes

For the past 40 years, catecholamines have been used for inotropic/lusitropic support of failing myocardial function with poor cardiac output. Because of the risk of complications, this treatment usually requires the patient to be in hospital and under conditions of cardiac monitoring. We describe the case of a patient in New York Heart Association (NYHA) class IV heart failure following heart transplantation who gained clinical benefit from inotrope treatment over a long period, both in an ambulatory mode and home environment.

Case report

A 49 year old man with heart failure due to ischaemic heart disease had orthotopic heart transplantation in July 1993. He had had a large inferior myocardial infarction eight years earlier, followed three years later by a triple vessel coronary artery bypass grafting. $\mathrm{He}$ deteriorated steadily in the four years after his bypass grafting. Immediately before transplant surgery, he had an exercise tolerance of less than 100 yards. The donor heart was from a 29 year old woman of $65 \mathrm{~kg}$ body weight (the recipient weighed $75 \mathrm{~kg}$ ). She was a life long non-smoker who had a fatal trauma to the head as a result of a traffic accident. The total ischaemic time was three hours 40 minutes and initially there was good function. However, before the chest was closed, the heart was seen to be dilating. Inotropic support was started, and an intra-aortic balloon pump was inserted and maintained for three days before both could be discontinued with stable haemodynamics.

For the first two years after the heart transplant, the patient remained healthy on conventional triple therapy immunosuppression (prednisolone, cyclosporin, and azathioprine). The cardiac biopsies performed during this time showed no rejection, and were notable only for the presence of occasional huge cyclosporin nodules (Quilty lesions). Coronary angiography, performed at 12 and 24 months, showed normal haemodynamic values and systolic left ventricular function, with no evidence of coronary artery disease.

At 30 months, the patient complained of nausea and palpitations. Echocardiography showed that left ventricular ejection fraction had fallen from $70 \%$ to $54 \%$ (fig 1), and cardiac biopsy was performed for suspected cardiac rejection. Presumptive treatment was initiated with pulsed methylprednisolone $(1 \mathrm{~g}$ daily for three days) and, because of clinical deterioration, antithymocyte globulin was also given (100 mg daily for three days). Right ventricular biopsy showed a multifocal lymphoid cell interstitial infiltrate with some myocytolysis. Coronary angiography still showed no significant coronary artery disease and, after treatment, cardiac biopsies had cleared. However, the patient's exercise tolerance continued to deteriorate. Thirty two months after transplantation, exercise tolerance was reduced to 50 yards and the patient started taking an angiotensin converting enzyme inhibitor (captopril; Squibb, Hounslow, UK; $12.5 \mathrm{mg}$ three times daily). An initial improvement in left ventricular ejection fraction was followed by a
Accepted for publication 3 November 1998 


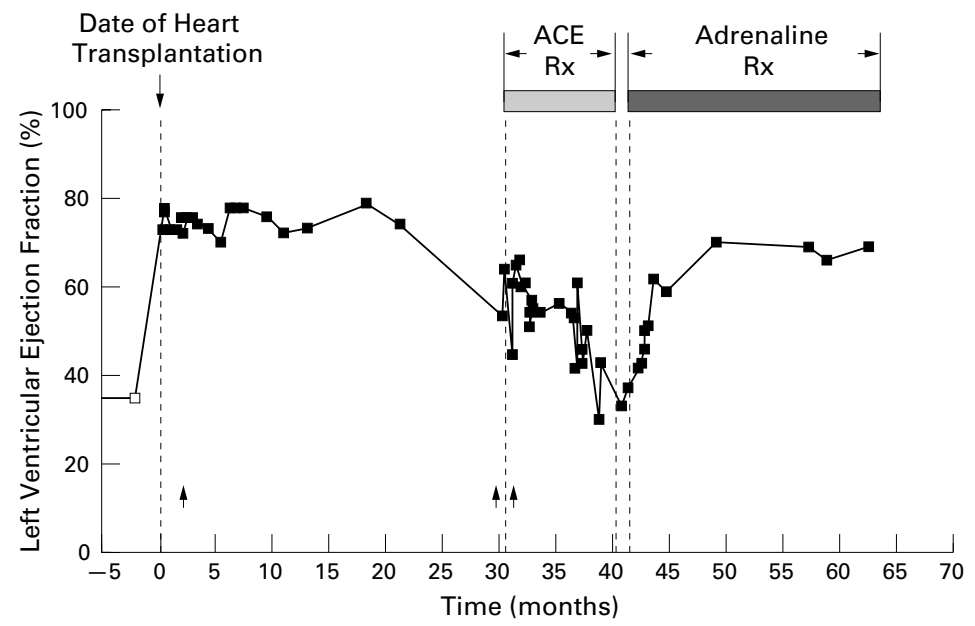

Figure 1 Left ventricular ejection fraction is shown with time from the date of heart transplantation. Note that after 20 months of intravenous adrenaline, the left ventricular. systolic function has returned almost to the baseline value immediately after transplantation. The bold vertical arrows indicate times of augmented immunosuppression with pulsed three day courses of intravenous methylprednisolone (1 g daily).

further decline in systolic performance, with echocardiographic determination of ejection fraction being as low as $37 \%$ (fig 1). At 38 months the patient was confined to a wheelchair as he was in heart failure with low cardiac output and dyspnoea at rest. He also had orthopnoea and required intravenous diuretics. The chest radiograph at this time is shown in fig 2A. A right heart catheter showed a mean right atrial pressure of $8 \mathrm{~mm} \mathrm{Hg}$, pulmonary wedge pressure of $28 \mathrm{~mm} \mathrm{Hg}$, pulmonary artery pressure of $44 / 20 \mathrm{~mm} \mathrm{Hg}$ with a mean of $32 \mathrm{~mm} \mathrm{Hg}$, and cardiac output of 1.2 litres/ min. Renal function had deteriorated and, despite a dopamine infusion, the creatinine clearance was only $40 \mathrm{ml} / \mathrm{min}$. Systolic blood pressure was low $(90 / 60 \mathrm{~mm} \mathrm{Hg})$ and in November 1996 he was given intravenous adrenaline in addition to dopamine. In the two weeks after this deterioration, the patient could not be weaned off the inotropic support and a Hickman line was inserted for long term drug delivery. After four weeks of hospital observation and adjustments to the inotrope infusion rates, the patient was weaned off dopamine and discharged home on an adrenaline infusion. Assessment while he was in hospital included continuous ECG monitoring, which was followed by 24 hour ambulatory ECG recording to exclude any proarrhythmic effects.

The drug delivery system for outpatient treatment was a CADD-1 battery powered external ambulatory pump system for continuous infusion (Simcare Ltd, St Pauls, Minnesota, USA; Southern Syringe Services, Southgate, London, UK) (fig 3). The reservoir has a $100 \mathrm{ml}$ capacity, which is filled by a local hospital pharmacy. The pump and reservoir are primed with $110 \mathrm{ml}$ of $5 \%$ glucose and $11 \mathrm{ml}$ of 1:1000 strength adrenaline which allows for excess. The infusion rate was $2 \mathrm{ml}$ hourly, which is equivalent with this dilution, at current body weight, to $38 \mathrm{ng} / \mathrm{kg} / \mathrm{min}$. At this rate of infusion, the reservoir provides inotropic support for a minimum of 48 hours, and the pump has an indicator to warn the patient when it needs to be refilled. Delivery to a central vein is via a tunnelled central venous Hickman line, sited under local anaesthesia, and with antibiotic cover.

The ejection fraction improved after one year of inotrope treatment, and the patient felt much better. Although he still had an exercise tolerance of only a few metres, the patient was no longer short of breath at rest, and had no dependent oedema. At most recent follow up in late 1998 he was comfortable at rest, had no ankle oedema, and a systolic blood pressure of $110 \mathrm{~mm} \mathrm{Hg}$. The current chest radiograph is shown in fig $2 \mathrm{~B}$. Renal function has improved with a creatinine clearance of $68 \mathrm{ml} / \mathrm{min}$. A right heart catheter at this time shows a mean pressure in the right atrium of $8 \mathrm{~mm} \mathrm{Hg}$, pulmonary artery wedge of $8 \mathrm{~mm} \mathrm{Hg}$, pulmonary artery pressure of $32 / 14 \mathrm{~mm} \mathrm{Hg}$ with a mean of $20 \mathrm{~mm} \mathrm{Hg}$, and cardiac output of 5.2 litres/min. Discussion with the patient about the risks associated with a second heart transplant, ${ }^{1}$ coupled with the patient's perception of an acceptable quality of life on inotropic support, resulted in a decision to remove the patient from the transplant waiting list and for him to continue on home inotropic support.

Recently, we conducted a trial withdrawal period from the adrenaline treatment the patient had been receiving for the previous 22 months. He was recumbent and with constant echocardiography and Doppler monitoring. Systolic arterial blood pressure fell marginally, but the calculated left ventricular ejection fraction and cardiac output dropped from baseline values of $72 \%$ and 4.2 litres/min to $59 \%$ and
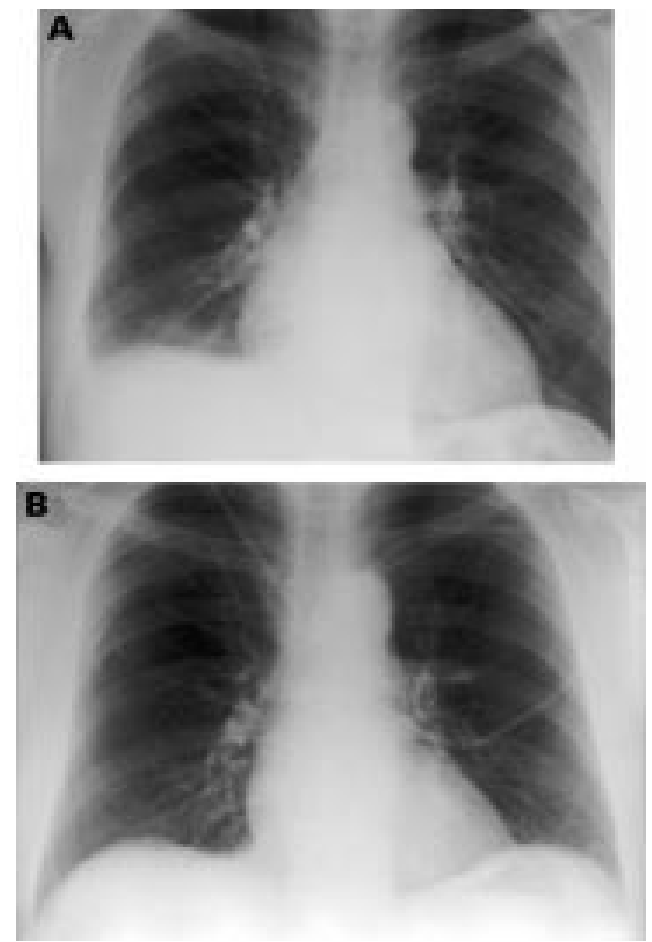

Figure 2 (A) Chest radiograph at 38 months (upper) when left ventricular function had fallen to its lowest value (ejection fraction 37\%); heart size is enlarged (cardiothoracic ratio 17/32) and there is evidence of pulmonary vascular congestion. (B) Most recent film (lower) shows a reduction in heart size (cardiothoracic ratio 15/32) and an improvement in pulmonary vascularity. 


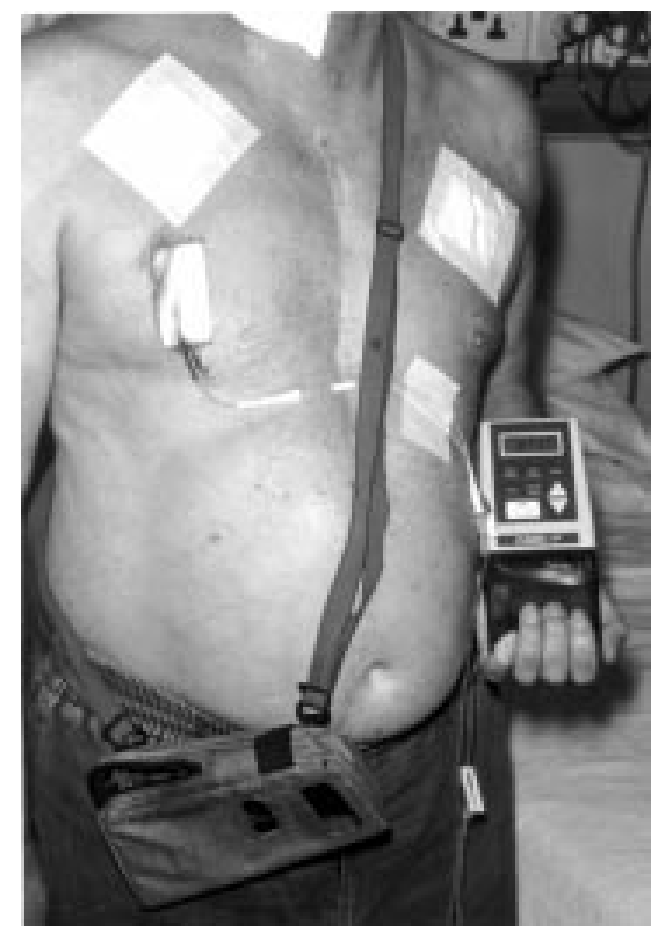

Figure 3 CADD-1 battery powered pump for administration of adrenaline shown in the patient's left hand, with holster for this pump on right side. The patient has just undergone replacement of the Hickman line (dressings on right side of chest) due to fracture of the previous line at the skin entry site. The dressing on the right side of neck was for the temporary placement of an internal jugular line and that on the left side of the chest covers the site of the previous Hickman line insertion.

3.1 litres/min, respectively. The most notable change was a prolongation of isovolumic relaxation time, from $65 \mathrm{~ms}$ at baseline to $90 \mathrm{~ms}$ at 35 minutes, which suggests slower ventricular relaxation. Within minutes of discontinuing the adrenaline, the patient felt hot and flushed. After 10 minutes, fatigue was the dominant symptom. These symptoms increased in intensity for 20 minutes and lasted until adrenaline was restarted after 35 minutes. Approximately one hour after returning to inotrope support, the patient felt completely back to normal.

\section{Discussion}

This case shows that continuous intravenous inotrope therapy is a practical option for treatment in the home; it can also have a beneficial clinical effect in an appropriately selected case. In our patient, cardiac failure developed to the point where oral treatment was no longer effective and inotropic support was needed. The options at that stage were prolonged hospitalisation, use of mechanical circulatory support, or home inotrope treatment. Our patient has experienced an improved quality of life on inotropic support, such as also been experienced by patients in end stage heart failure treated with dobutamine,,$^{2-4}$ and patients on the waiting list for heart transplantation. ${ }^{5}$

The risk of serious arrhythmia is a concern when deciding to initiate inotropic treatment. A high mortality rate has been reported in patients receiving intermittent dobutamine infusions for chronic heart failure ${ }^{6}$ However, in a patient already "inotrope dependent", this risk is negated as it is expected that the patient will die of circulatory failure without this treatment.

The use of oral inotropes, particularly phosphodiesterase inhibitors, has raised ethical issues of quality versus quantity of life. The medicolegal position surrounding outpatient home inotrope prescription is a problem, as informed consent is required from the patient. The treatment should be fully and carefully described and the patient should be informed of the disadvantages (risk of infection, complications surrounding line placement, rhythm disturbance, and sudden death) as well as the potential benefits (particularly discharge from hospital); this process should be documented in the patient's records. The patient's general practitioner should be consulted early in the process to establish their willingness to participate in the supervision. Monitoring electrolyte balance to reduce the risk of arrhythmia is another problem. In our case, this was facilitated by the need for regular blood tests to measure blood cyclosporin and its effect on renal and hepatic function. Arrangements for provision of drugs should also be made with a hospital pharmacy local to the patient. The treatment can be further complicated, for example-while he has been on inotrope treatment, our patient has experienced two fractures of the venous access line, both of which required hospital admission to change the line.

The economic impact of heart failure is massive and evidence suggests that the reduction of costs from this form of treatment are significant when compared to the costs of hospitalisation. ${ }^{7}$ The current price of the CADD-1 pump used by our patient (including the pump pouch) is $£ 1905$ (US\$3095), and medication cassette reservoirs of $100 \mathrm{ml}$ capacity cost $£ 226$ (US\$367) for 12 . Additionally, home intravenous inotrope treatment has been shown to reduce the length of stay in hospital, the number of hospital admissions, and the improved functional class of patients with advanced heart failure (NYHA class IV). ${ }^{7}$

A transplanted donor heart is surgically denervated and therefore may exhibit supersensitivity to $\beta$ adrenergic agonists. A recent study suggests that there is no evidence for an increase in $\beta$ adrenergic receptor density or supersensitivity of postsynaptic origin in human orthotopic cardiac allografts. However, such hearts do exhibit $\beta$ adrenergic supersensitivity of the sinus node (presynaptic in origin) which should increase the response of the transplanted heart to circulating adrenaline or noradrenaline (norepinephrine). ${ }^{8}$ The mechanisms of receptor activity in our patient are beyond the scope of this report, but we have encountered no problems with either supersensitivity to adrenaline or the development of tachyphylaxis to continuous infusion.

We believe that home inotrope treatment is a reasonable therapeutic option for patients who are not eligible for heart transplantation. When selecting a patient, there should be no obvious predilection to arrhythmia or ongoing 
ischaemia, a satisfactory psychosocial situation, and a team approach to outpatient care.

1 Hosenpud JD, Bennett LE, Keck BM, et al. The Registry for the International Society for Heart and Lung Transplantathe International Society for Heart and Lung Transplantation: fifteenth

2 Applefeld MM, Newman KA, Grove WR, et al. Intermittent, continuous outpatient dobutamine infusion in the management of congestive heart failure. Am $\mathcal{f}$ Cardiol 1983;51:455-8

3 Miller LW, Merkle EJ, Hermann V. Outpatient dobutamine for end stage congestive heart failure. Crit Care Med 1990; 18:S30-3.
4 Miller LW. Outpatient dobutamine for refractory congestive heart failure: advantages, techniques and results. F Heart heart failure: advantages, techniq
Lung Transplant 1991;10:482-7.

5 Miller LW, Merkle EJ, Jennison SH. Out patient use of dobutamine to support patients awaiting heart transplantation. f Heart Lung Transplant 1993;13:S126-9.

6 Dies F. Intermittent dobutamine in ambulatory patients with chronic cardiac failure. Br f Clin Pract 1986;40(suppl): $37-9$.

7 Harjai KJ, Mehra MR, Ventura HO, et al. Home inotropic therapy in advanced heart failure. Chest 1997;112:1298303 .

8 Gilbert EM, Eiswirth CC, Mealy PC, et al. Beta-adrenergic supersensitivity of the transplanted human heart is presynaptic in origin. Circulation 1989;79:344-9.

\title{
Familial fibromuscular dysplasia of bilateral brachial arteries
}

\author{
H Suzuki, H Daida, H Sakurai, H Yamaguchi
}

\section{Department of Cardiology, Juntendo University School of Medicine, 2-1-1 Hongo, Bunkyo-ku, Tokyo, 113-8421, Japan H Suzuki \\ H Daida \\ H Sakurai \\ H Yamaguchi \\ Correspondence to: Dr Suzuki. \\ e-mail: hisuzuki@ med.juntendo.ac.jp

A 65 year old housewife presented to our hospital with occasional left chest pains at rest. Bruits were heard from the bilateral carotid, brachial, and lower abdominal area. All arterial pulses of the extremities were noted to be normal and there was no coldness, cyanosis, or ulcer formation of fingers and toes. Although an ECG showed incomplete right bundle block and the Master's exercise tolerance test was negative, coronary angiography was performed.

Angiography of the right axillary brachial artery was done before coronary angiography because it was difficult to insert a catheter through the right brachial artery. The angiogram showed the characteristic appearance of fibromuscular dysplasia (fig 1). Coronary arteriograms revealed tortuous but normal coronary arteries (fig 2).

An abdominal aortography also showed long segmental string of beads appearance of

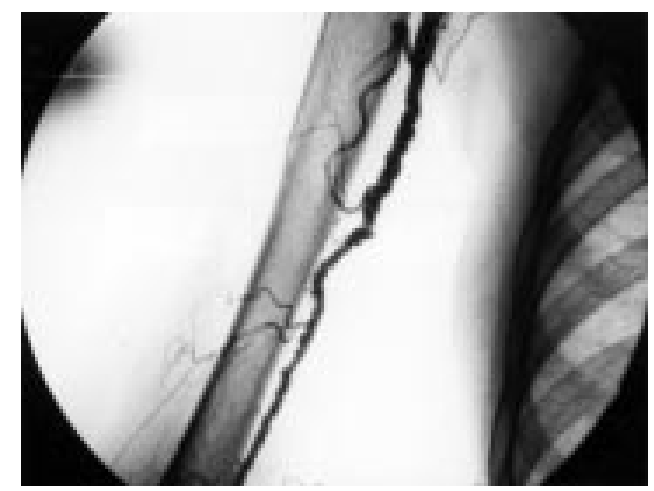

Figure 1 Angiogram of the right midbrachial artery shows the long segmental string of beads appearance characteristic of fibromuscular dysplasia. bilateral external and internal iliac arteries with symmetrical involvement (fig 3). Moreover, the bilateral internal carotid, external carotid, and vertebral arteries were segmentally involved in fibromuscular dysplasia. An angiography of the left upper extremities showed the same angiographic appearance, similar to the lesion of the right brachial artery. Systemic fibromuscular dysplasia was finally diagnosed. The patient denied either tobacco abuse or traumatic accidents. She had no history of cerebral stroke or immunological collagen diseases.

The patient's 89 year old mother, who had complained of coldness of fingers and toes, especially in winter, for several decades, also had bilateral brachial and abdominal bruits. Brachial arteriographies demonstrated focal segmental beadings with narrowing of the bilateral brachial arteries. The angiographic findings are compatible with fibromuscular dysplasia of the brachial arteries. The patient's grandfather, uncle, and male cousin died of cerebral infarction, the last from a stroke at a young age.

Mettinger et al extensively reviewed and classified over 1100 cases of fibromuscular dysplasia by location in $1982 .{ }^{1}$ The renal artery was involved in $58 \%$ of patients, the internal carotid and vertebral artery in $32 \%$, the iliac or external artery in approximately $2 \%$ of patients, and the coeliac or mesenteric artery in $1 \%$ of patients. The first case of brachial artery fibromuscular dysplasia was reported by Keßler in $1982^{2}$ and subsequently 11 other cases have been described; however, there are no reports of familial occurrence of bilateral brachial artery fibromuscular dysplasia. 

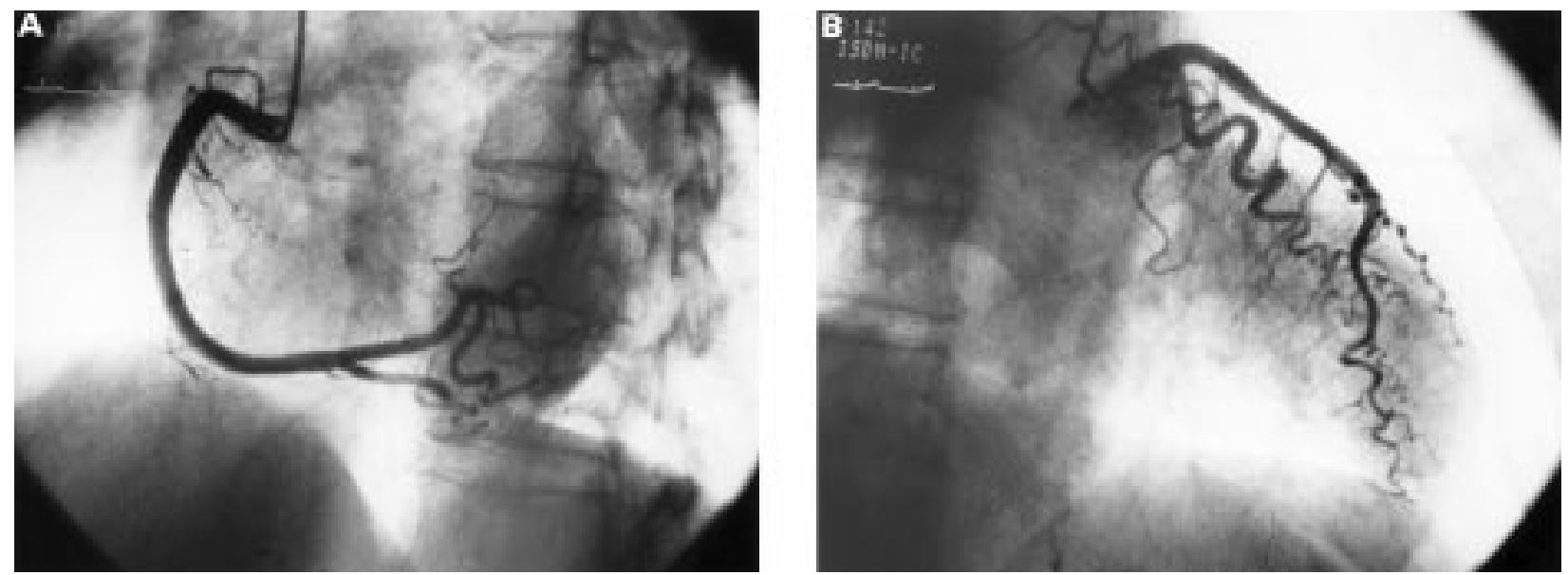

Figure 2 Coronary arteriograms showing normal coronary vessels that are tortuous without involvement of fibromuscular dysplasia. (A) right coronary artery; (B) left coronary artery.

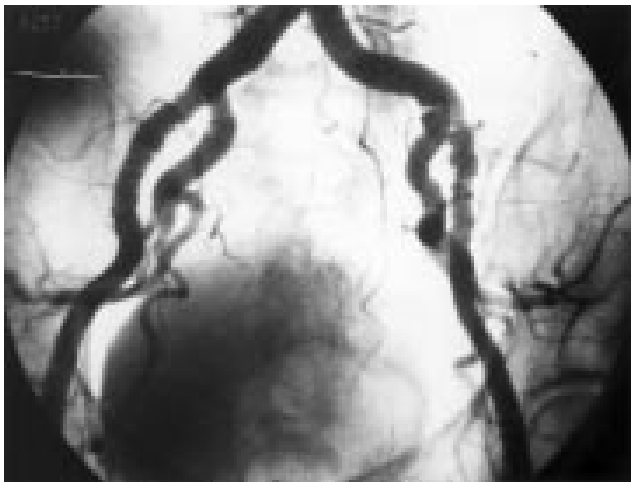

Figure 3 An abdominal aortogram shows the string of beads appearance characteristic of fibromuscular dysplasia in bilateral external and internal iliac arteries. The

involved lesions of the bilateral iliac arteries are completely symmetrical.

The aetiology of fibromuscular dysplasia is not well understood, and several hypotheses have been proposed ${ }^{3}$; hormonal factors, mechanical trauma, a genetic predisposition, metabolic and immunological factors, as well as intrinsic deficiency of elastic fibres. Fibromuscular dysplasia may represent an acquired developmental vascular disorder affected by various intrinsic and extrinsic factors in patients with hereditary abnormalities of the arterial walls. Several observations of the occurrence of fibromuscular dysplasia in one family ${ }^{5}$ as well as the present case, strongly support the concept of a genetically transmitted abnormality. Clinical studies analysing the pedigree of several families with fibromuscular dysplasia, suggest that in some cases it is inherited as an autosomal dominant trait with reduced penetrance in males. ${ }^{134}$

In conclusion, we report the findings of fibromuscular hyperplasia of the bilateral brachial arteries in mother and daughter with a family history of cerebrovascular complications. This supports the concept of a genetic abnormality, although familial occurrence of fibromuscular dysplasia is extremely rare. Moreover, the distribution of complete symmetrical involvements of multifocal fibromuscular dysplasia lesions in these cases suggest the possible hereditary nature of the disease.

1 Mettinger KL. Fibromuscular dysplasia and the brain: current concept of the disease. Stroke 1982;13:53-8.

2 Keßler M. Fibromuskuläre Dysplasie der A brachialis. Radiologe 1982;22:185-7.

3 Lüscher TF, Lie JT, Stanson AW, et al. Arterial fibromuscular dysplasia. Mayo Clin Proc 1987;62:931-52.

4 Mettinger KL, Ericson K. Fibromuscular dysplasia and the brain: observations on angiographic, clinical and genetic characteristics. Stroke 1982;13:46-52.

5 Halpern MM, Sanford HS, Viamonte M Jr. Renal-artery abnormalities in three hypertensive sisters: probable famil 